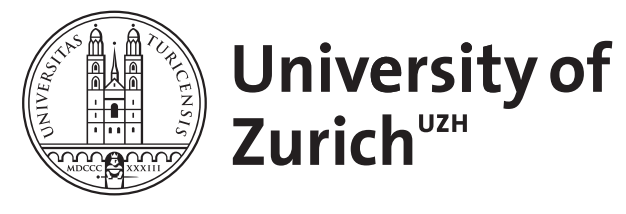

\title{
Kontrazeption bei Frauen mit Migräne
}

\author{
Merki-Feld, G S
}

\begin{abstract}
Die Migräne ist charakterisiert durch wiederkehrende, starke bis invalidisierende, meist einseitige Schmerzattacken, welche teilweise von Übelkeit, Erbrechen und sensorischer Überempfindlichkeit begleitet sind. Epidemiologische Studien zeigen, dass 12 Prozent der Bevölkerung betroffen sind, Frauen häufiger als Männer (Relation: 3:1) (1). Der Beginn der Erkrankung liegt typischerweise vor dem 40. Lebensjahr. Die Diagnose wird klinisch gestellt entsprechend den Kriterien der International Headache Society (Tabelle 1). Die Pathophysiologie der Migräne ist komplex und wird heute erst teilweise verstanden. Genpolymorphismen spielen sicher eine Rolle, ebenso vaskuläre und hormonale Faktoren (1-3). Die Hauptursache und das auslösende Ereignis sind jedoch unbekannt. Daher basiert die Diagnose auf den berichteten Symptomen. Die International Headache Society (IHS) klassifiziert die Migräne in zwei Untertypen: - Migräne mit Aura und - Migräne ohne Aura.
\end{abstract}

Posted at the Zurich Open Repository and Archive, University of Zurich ZORA URL: https://doi.org/10.5167/uzh-29398

Journal Article

Accepted Version

Originally published at:

Merki-Feld, G S (2009). Kontrazeption bei Frauen mit Migräne. Ars Medici III:37-39. 


\title{
Kontrazeption bei Frauen mit Migräne
}

Welche Methoden sind geeignet?

\begin{abstract}
Die Migräne ist eine komplexe invalidisierende Erkrankung, welche überwiegend Frauen im reproduktiven Alter betrifft. Ziel bei betroffenen Frauen muss es sein, eine Verhütungsmethode zu finden, die weder mit einem erhöhten Insultrisiko assoziiert ist noch den Migräneverlauf negativ beeinflusst. Im Folgenden werden diagnostische Voraussetzungen, Ausschlusskriterien und Empfehlungen für die Methodenwahl mit reinen Gestagenpräparaten gegeben.
\end{abstract}

\section{Gabriele Merki}

Die Migräne ist charakterisiert durch wiederkehrende, starke bis invalidisierende, meist einseitige Schmerzattacken, welche teilweise von Übelkeit, Erbrechen und sensorischer Überempfindlichkeit begleitet sind. Epidemiologische Studien zeigen, dass 12 Prozent der Bevölkerung betroffen sind, Frauen häufiger als Männer (Relation: 3:1) (1). Der Beginn der Erkrankung liegt typischerweise vor dem 40. Lebensjahr. Die Diagnose wird klinisch gestellt entsprechend den Kriterien der International Headache Society (Tabelle 1).

Die Pathophysiologie der Migräne ist komplex und wird heute erst teilweise verstanden. Genpolymorphismen spielen sicher eine Rolle, ebenso vaskuläre und hormonale Faktoren (13). Die Hauptursache und das auslösende Ereignis sind jedoch unbekannt. Daher basiert die Diagnose auf den berichteten Symptomen. Die International Headache Society (IHS) klassifiziert die Migräne in zwei Untertypen:

- Migräne mit Aura und

- Migräne ohne Aura.

Migränikerinnen mit Aura erleben zusätzlich fokale neurologische Symptome, die meist dem starken Kopfschmerz vorangehen.

Migräne, zerebraler Insult und hormonale Therapie

Ein wichtiger Aspekt für die hormonale Therapie der Frauen mit Migräne ist der in vielen Studien gezeigte Zusammenhang zwischen Migräne und Hirninfarkt, welcher durch die Einnahme ethinylestradiolhaltiger Kontrazeptionspräparate potenziert werden kann (4-9). Die Migräne mit Aura stellt einen grösseren Risikofaktor für einen Hirninfarkt dar als die Migräne ohne Aura (Tabelle 2).

Ischämische Infarkte treten meistens nicht während, sondern zwischen den Migräneattacken auf. Der Pathomechanismus, der hier eine Rolle spielt, wird immer noch diskutiert. Ein offenes Foramen ovale wird gehäuft gefunden bei Frauen mit Auramigräne und ist ein Risikofaktor für einen ischämischen Infarkt (10). Der Verschluss des Foramen ovale hat teilweise zu einer Reduktion der Migräneanfälle geführt.

\section{Prävalenz von Migräne und Östrogenexposition}

Es ist bekannt, dass Hormonschwankungen im Zyklus der Frau Kopfschmerzen beeinflussen können. Die Prävalenz der Migräne nimmt zu bei Mädchen von der Adoleszenz bis zum frühen Erwachsenenalter (11). Viele Frauen erleiden Attacken im Zusammenhang mit der Menstruation. Dagegen hat die Schwangerschaft in der Regel einen positiven Einfluss, hier kommt es seltener zu Migräneattacken (12). In der Menopause kommt es häufig zu einem Rückgang der Migräneanfälle, wogegen eine Hormonersatztherapie zur erneuten Exazerbation der Migräne führen kann (13). 
Heute gibt es plausible Erklärungen für den Zusammenhang zwischen Migräne und Östrogenen. Östrogenrezeptoren finden sich in vielen Hirnarealen. Interaktionen dieser Rezeptoren mit Neurotransmittersystemen beeinflussen die Steuerung der Kaliumpermeabilität auf der Ebene von Ionenkanälen sowie die Freisetzung verschiedener mit der Migräne assoziierter Neurotransmitter (1). Darüber hinaus sind bestimmte Polymorphismen des Östrogen- und Progesteronrezeptors mit einer Verdopplung des Migränerisikos assoziiert (2). Tabelle 3 gibt eine Übersicht über die Formen der hormonassoziierten Migränen.

\section{Migränerisiko unter kombinierten Antikonzeptiva und reinen Gestagenpräparaten}

Ziele der Kontrazeption bei Migränikerinnen sind einerseits die Verhinderung eines weiteren Anstiegs des Insultrisikos und andererseits die Vermeidung einer Verschlechterung des Migräneleidens. Ethinylestradiolhaltige Kontrazeptiva führen bei Migränikerinnen zum Anstieg des Insultrisikos. Dies ist bei reinen Gestagenpräparaten oder Intrauterinpessaren nicht der Fall.

Das Insultrisiko unter Einnahme von Kombinationspillen variiert in Abhängigkeit vom Migränetyp und hängt ferner davon $a b$, ob weitere Risikofaktoren vorliegen (bei jungen Frauen ist das Rauchen an erster Stelle der Risikofaktoren zu nennen) (Tabelle 2). Darüber hinaus können kombinierte Kontrazeptiva eine Migräne verändern oder neu verursachen: So kommt es vor, dass Migräneattacken erstmals auftreten, eine vorbestehende Migräne häufiger wird oder der Hormonentzug in der Pillenpause Kopfschmerzen oder auch Migräneattacken auslöst (14). In retrospektiven Studien führt die Anwendung kombinierter Pillenpräparate bei Migränikerinnnen in 24 bis 35 Prozent zu einer Verschlechterung und nur bei 5 Prozent zu einer Verbesserung der Symptome (14). Eine kürzlich publizierte grosse Populationsstudie zur Untersuchung der Prävalenz von Kopfschmerzen ergab, dass die Anwendung niedrig dosierter Kombinationspräparate assoziiert ist mit einem erhöhten Risiko für sowohl Kopfschmerzen als auch für Migräne. Dagegen zeigte sich für Gestagenpräparate kein erhöhtes Risiko $(14,15)$.

Medikamente können auch als Trigger für eine Migräne fungieren. Deshalb ist bei der Anwendung von Gestagenpräparaten zu empfehlen, die Verträglichkeit bei oraler Applikation zu testen, bevor Implantate oder Depotpräparate eingesetzt werden. Gestagenpräparate zur Ovulationshemmung werden im Gegensatz zur kombinierten Pille immer kontinuierlich angewandt. Somit entfällt der Hormonentzug, der bei Migränikerinnen mit kombinierter Pille, aber auch im normalen Zyklus ein häufiger Trigger ist. Ovulationshemmende Gestagene sind daher nicht nur zur Verhütung, sondern auch therapeutisch bei menstrueller Migräne einsetzbar.

\section{Empfehlungen}

Der Bedarf nach sicherer Verhütung sollte keiner Patientin gesundheitlich schaden. Ziel bei Frauen mit Migräne muss es sein, eine Verhütungsmethode zu finden, die weder assoziiert ist mit einem erhöhten Insultrisiko noch denMigräneverlauf negativ beeinflusst. Hier empfiehlt es sich, in drei Schritten vorzugehen:

- An erster Stelle stehen die genaue Diagnostik und Einordnung der Kopfschmerzen (Differenzialdiagnose Kopfschmerzen, Migräne ohne Aura, Migräne mit Aura). Hierzu bieten die Tabellen eine Hilfe. Gelegentlich ist es erforderlich, einen Spezialisten zurate zu ziehen. - Die nächsten Schritte sind die Anamnese und Untersuchung zum Ausschluss weiterer kardiovaskulärer Risikofaktoren.

- Im dritten Schritt ist es sinnvoll zu verifizieren, ob die Migräne zyklusabhängig ist, da in solchen Fällen eine ununterbrochene Gestageneinnahme für den Migräneverlauf von therapeutischem Nutzen ist. 
Zum Vorgehen vergleiche Tabelle 4.

\section{Zusammenfassung}

Die Migräne ist eine komplexe invalidisierende Erkrankung, welche überwiegend Frauen im reproduktiven Alter betrifft. Kombinierte Pillen können die Migräne verstärken und erhöhen das Risiko für einen zerebralen Insult multiplikativ. Daher sind bei Migränikerinnen ethinylestradiolfreie Verhütungsmethoden indiziert. Ovulationshemmende Gestagenmethoden haben häufig durch die ununterbrochene Einnahme einen positiven Effekt auf die Kopfschmerzen, ohne das Risiko für einen zerebralen Insult zu erhöhen. Unter Beachtung der üblichen Kontraindikationen können auch Intrauterinpessare empfohlen werden.

Dr. med. Gabriele Susanne Merki

Leiterin Zentrum für Schwangerschaftsverhütung

UniversitätsSpital Zürich

Frauenklinikstrase 10

8091 Zürich

E-Mail: gabriele.merki@usz.ch

Quellen:

1. Martin VT, Behbehani M.: Ovarian hormones and migraine headache: understanding mechanisms and pathogenesis — part I. Headache 2006; 46: 3-23.

2. Colson NJ, Lea RA, et al.: The role of vascular and hormonal genes in migraine susceptibility.

Mol Genet Metab 2006; 88: 107-113.

3. Gupta S, Mehrotra S, et al.: Potential role of female sex hormones in the pathophysiology of migraine. Pharmacol Ther 2007; 113: 321-340.

4. Kurth T.: Migraine and ischaemic vascular events. Cephalalgia 2007; 27: 965-975.

5. Tzourio C, Iglesias S, et al.: Migraine and risk of ischaemic stroke: a case-control study. BMJ 1993; 307: 289-292.

6. Tzourio $\mathrm{C}$, Tehindrazanarivelo A, et al.: Case-control study of migraine and risk of ischaemic stroke in young women. BMJ 1995; 310: 830—833.

7. Bousser MG, Welch KM.: Relation between migraine and stroke. Lancet Neurol 2005; 4: $533-542$.

8. Chang CL, Donaghy M, Poulter N.: Migraine and stroke in young women: casecontrol study. The World Health Organisation Collaborative Study of Cardiovascular Disease and Steroid Hormone Contraception. BMJ 1999; 318: 13-18.

9. Etminan M, Takkouche B, et al.: Risk of ischaemic stroke in people with migraine: systematic review and meta-analysis of observational studies. BMJ 2005; 330: 63.

10. Wilmshurst P, Nightingale S.: Relationship between migraine and cardiac and pulmonary right-to-left shunts. Clin Sci 2001; 100: 215-220.

11. MacGregor EA.: Female sex hormones and migraine. Rev Neurol 2005; 161: 677678.

12. MacGregor EA.: Menstruation, sex hormones, and migraine. Neurol Clin 1997; 15: $125-141$.

13. MacGregor EA.: Migraine and the menopause. J Br Menopause Soc 2006; 12: 104 108.

14. Aegidius K, Zwart JA, et al.: Oral contraceptives and increased headache prevalence: the Head-HUNT Study. Neurology 2006; 66: 349-353. 
15. Wober C, Brannath $\mathrm{W}$, et al.: Prospective analysis of factors related to migraine attacks:

the PAMINA study. Cephalalgia 2007; 27: 304-314.

16. Bousser MG, Conard J, et al.: Recommendations on the risk of ischaemic stroke associated with use of combined oral contraceptives and hormone replacement therapy in women with migraine. The International Headache Society Task Force on Combined Oral Contraceptives \& Hormone Replacement Therapy. Cephalalgia 2000; 20: 155-156.

\section{Tabelle 1: Diagnosekriterien für Migräne mit und ohne Aura}

\section{Migräne ohne Aura}

A Mindestens 5 Attacken, welche die Kriterien B bis D erfüllen

B Kopfschmerzdauer 4 bis 72 Stunden

C Mindestens 2 der folgenden Charakteristika müssen erfüllt sein:

$\mathrm{n} \quad$ Einseitige Lokalisation

n Pulsierender Charakter

n Mittlere oder starke Schmerzintensität

n Verstärkung durch tägliche körperliche Routineaktivitäten

D Mindestens eines der folgenden Symptome ist vorhanden:

n Übelkeit und/oder Erbrechen

n Photophobie und Phonophobie

E Eine andere Kopfschmerzursache kann ausgeschlossen werden

\section{Migräne mit Aura}

A Mindestens 2 Attacken, welche die Kriterien B bis D erfüllen

B Die Aura besteht aus mindestens einem der folgenden Symptome:

n Vollständig reversible visuelle Symptome

n Vollständig reversible dyspathische Symptome

C Mindestens 2 der folgenden Punkte sind erfüllt:

$\mathrm{n} \quad$ homonyme visuelle Symptome und/oder einseitige sensible Symptome

$\mathrm{n} \quad$ wenigstens 1 Aurasymptom entwickelt sich allmählich $\geq 5$ Minuten hinweg und/oder verschiedene Aurasymptome in Abständen von 5 Minuten

$\mathrm{n} \quad$ jedes Symptom hält $\geq 5$ Minuten und $\leq 60$ Minuten an

D Kopfschmerzen beginnen noch während der Aura oder folgen ihr innerhalb von 60 Minuten

E Eine andere Kopfschmerzursache kann ausgeschlossen werden

Adaptiert entsprechend der IHS-II-Klassifikation

\section{Tabelle 2: Migräne und Insultrisiko für Frauen Alter < 45 Jahre}

Risiko Odds Ratio für zerebralen Insult

Migräne $\quad 1,5-2,3$

Migräne mit Aura $\quad 3,8-8,4$ 
Migräne ohne Aura 1,1-2,9

Migräne und Rauchen $\quad 10$

Migräne und kombinierte Pille 5-17

Migräne, kombinierte Pille, Rauchen 13,9-34,2

Modifiziert nach IHS, Cephalalgia 2000, 155-156. (16)

Tabelle 3: Kriterien für hormonassoziierte Kopfschmerzen gemäss der Internationalen Kopfschmerzklassifikation

Reine menstruelle Migräne ohne Aura

1. Keine Aura

2. Migräneattacken nur an den Zyklustagen -2 bis +3 und zu keiner anderen Zeit des Zyklus

Menstruationsassoziierte Migräne

1. Keine Aura

2. Migräneattacken an den Zyklustagen -2 bis +3 und auch zu anderen Zeiten des Zyklus

Hormoninduzierte Migräne

1. Regelmässige Hormoneinnahme (Kontrazeption/Hormonersatztherapie)

2. Migräne/Kopfschmerz entwickelt sich innerhalb von 3 Monaten nach Einnahmebeginn

3. Migräne/Kopfschmerz bessert sich innerhalb von 3 Monaten nach Absetzen der

Hormone

Östrogenentzugskopfschmerz

1. Täglicher Gebrauch von Hormonen für mindestens 21 Tage, dann Unterbrechung

2. Migräne/Kopfschmerz entwickelt sich innerhalb von 5 Tagen nach letzter

Östrogeneinnahme

3. Migräne/Kopfschmerz legt sich innerhalb von 3 Tagen

Modifiziert nach IHS

Tabelle 4: Empfehlungen für die Kontrazeption je nach Migränetyp

1. Migräne ohne Aura

Keine weiteren Risikofaktoren: ovulationshemmende Gestagenpräparate* Intrauterinpessare

Relative Kontraindikation für kombinierte Pillen

Zusätzliche Risikofaktoren ovulationshemmende Gestagenpräparate* Intrauterinpessare

Absolute Kontraindikation für kombinierte Pillen

2. Migräne mit Aura ovulationshemmende Gestagenpräparate*

Intrauterinpessare

Absolute Kontraindikation für kombinierte Pillen

3. Menstruelle Migräne ovulationshemmende Gestagenpräparate*

(häufig Benefit für Migräneverlauf)

Intrauterinpessare

4. Pillenpausenkopfschmerz an pillenfreien Tagen Estradiol 1-2 mg oral

(Migräne) Estradiolpatch $50 \mu \mathrm{g} / 24$ Stunden

Wechsel auf Gestagenmethode* 
*Liste der in der Schweiz verfügbaren ovulationshemmenden Gestagenpräparate zur Kontrazeption

Oral

Cerazette ${ }^{\circledR} \quad$ Desogestrel, $75 \mu \mathrm{g} /$ kontinuierlich

Implantat

Implanon ${ }^{\circledR} \quad$ Etonogestrel $68 \mathrm{mg} / 3$ Jahre

Depotspritze

Depo-Provera ${ }^{\circledR} \quad$ Medroxyprogesteronazetat $150 \mathrm{mg}$ tief i.m. / 12-wöchentlich 